\title{
THE INFLUENCE OF ION BEAM MIXED Ni-Al SURFACE LAYERS ON FATIGUE IN POLYCRYSTALLINE NICKEL
}

\author{
D. S. Grummon and J. W. Jones \\ Department of Materials and Metallurgical Engineering \\ University of Michigan \\ Ann Arbor, MI 48109 USA \\ (Received July 18, 1985) \\ (Revised December $2 \dot{3}, 1985$ )
}

\begin{abstract}
Introduction
Though fatigue crack propagation is generally used as the basis for engineering design for fatigue, it is well established that up to $90 \%$ of fatigue life may be spent in the crack initiation phase. The phenomenon of fatigue crack initiation is known to be a highly surface sensitive process: cracks nucleate there, environmental attack is mediated there, and the egress of mobile dislocations is influenced by surface properties. It is natural, then, to look to surface treatments for means to improve the fatigue life of metals. Many previous approaches have employed modifications to the surface stress state or structure using mechanical methods like shot peening $(1,2)$ or by some metallurgical treatment, such as plating (3). Recently, the technique of ion implantation, now established as a commercial process for wear control, and which shows promise for use to impart corrosion resistance, is beginning to be evaluated for its potential application to fatigue problems (4-9).
\end{abstract}

When annealed FCC single crystals are cyclically loaded in plastic strain control, rapid dislocation multiplication gives rise to very high initial hardening rates (10), which eventually decline to zero at accumulated strains of ten or so, producing a plateau in the cyclic hardening curve at a stress level commonly called the saturation stress (11). The behavior of polycrystals in this respect is now thought to be generally analogous to the case for single crystals, though it is naturally more complex (12). Cyclic plastic straining is also known to produce intense strain localization in narrow lamellar bands of "soft" material, called persistent slip bands (PSB's), which invariably intersect the surface, producing intrusions and extrusions that are intimately associated with crack initiation processes (1315). The suppression of PSB formation, or the alteration of PSB action at the free surface, could thus be expected to have beneficial effects on fatigue crack initiation lifetimes. In the present study, the effect of an ion beam modified surface layer on fatigue crack initiation phenomena and cyclic plasticity is examined using pure polycrystalline nickel samples which have been implanted with aluminum using ion beam mixing. The results described here show that an ion beam mixed surface layer increases the saturation stress and tends to suppress the emergence of PSB's at the free surface of nickel samples cycled under plastic strain control. These effects are seen to be strongest for strain amplitudes in which PSB's are known to carry the bulk of the plastic strain during cycling.

\section{Experimental Procedures}

Fatigue specimens were machined from nickel alloy 270 to the geometry shown in Fig. 1 . Flat surfaces approximately $4 \mathrm{~mm}$ wide were machined on opposite sides of the gage section to facilitate optical micrographic observation of surface morphological changes during fatigue and to present a uniform incidence angle for the ion beam. After machining, the flat surface of each specimen was polished by hand using silicon carbide papers and 1 micron diamond paste. All of the specimens were then annealed in an atmosphere of dry hydrogen for three hours at $800^{\circ} \mathrm{C}$. The resulting grain size was 
approximately .3 to $1 \mathrm{~mm}$. The specimens were then electropolished using $20 \%$ perchloric acid in ethanol at $-40^{\circ} \mathrm{C}$.

FIG. 1

Axial fatigue specimen geometry. A flat surface was machined onto opposite sides of the guage section to facilitate surface study.

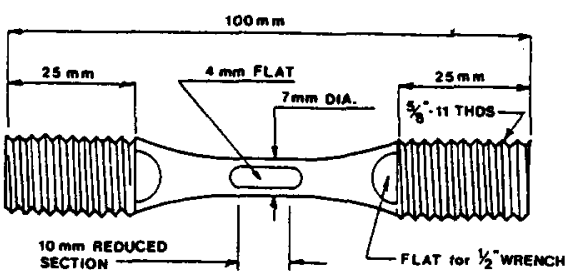

Surface modification by recoil ion implantation was carried out at the Tandem Accelerator Facility at Argonne National Laboratory. First, alternate layers of nickel and aluminum were deposited on the surface by vacuum evaporation at $2 \times 10^{-5} \mathrm{~Pa}$. Seven layers were applied as follows: $10.1 \mathrm{~nm} \mathrm{Ni}$, $9.6 \mathrm{~nm} \mathrm{Al}, 10.0 \mathrm{~nm} \mathrm{Ni}, 5.9 \mathrm{~nm} \mathrm{Al}, 11.4 \mathrm{~nm} \mathrm{Ni}, 5.4 \mathrm{~nm} \mathrm{Al}$, and finishing with $10 \mathrm{~nm}$ of nickel. After vacuum deposition, the specimens were promptly transferred to the accelerator target chamber and exposed to $0.5 \mathrm{MeV}$ krypton ions to a dose of $1 \times 10^{16} \mathrm{~cm}^{-2}$ at $5 \times 10^{-5} \mathrm{~Pa}$, to ballistically mix these

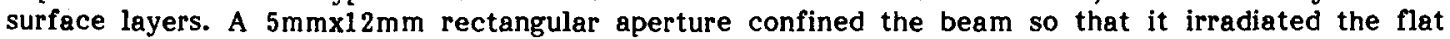
portion of the gage section, and each specimen was irradiated on both sides, such that about $30 \%$ of the total gage section area was treated.

Plastic strain controlled fatigue tests were carried out on a servo-hydraulic load frame using cast metal grips and a high sensitivity extensometer. A digital system was used which was capable of operation at $1 \mathrm{~Hz}$ while maintaining the plastic strain amplitude to within 1 or 2 percent of the target strain at plastic strain amplitudes as low as $3 \times 10^{-5}$ or as high as $7 \times 10^{-3}$, and which provided high quality cyclic hardening curves for saturation stress determination. Detailed hysteresis loops were acquired at lower frequencies $(.05-.1 \mathrm{~Hz}$.) and stored for subsequent analysis. In order to determine the effect of the ion beam mixed $\mathrm{Ni}-\mathrm{Al}$ surface layer on cyclic stress-strain behavior, several samples of both implanted and unmodified nickel were cycled at $1 \mathrm{~Hz}_{-3} \mathrm{~Hz}$ to total accumulated strains of 10 to 20 at plastic strain ranges of $3 \times 10^{-5}, 6.6 \times 10^{-5}, 3 \times 10^{-4}, 1 \times 10^{-3}, 3 \times 10^{-3}$, and $7 \times 10^{-2}$. Saturation stresses were determined and the cyclic stress strain curve was established for both modified and unmodified material. In some cases, several saturation stress values were determined from a single specimen using step tests.

In addition to mechanical property measurements, detailed observations were made of the evolution of surface slip and the development of surface morphology as a function of plastic strain accumulation. To track changes in surface features, single stage replicas were made of the flat surfaces of the specimens at frequent intervals during the fatigue test. In this way, the first emergence of persistent slip bands at the surface of the specimen could be determined as a function of accumulated strain. The degree of surface roughening and the density of slip bands was also compared between surface modified and unmodified specimens. Specimens were cycled to saturation and removed for detailed SEM observation of the surfaces.

\section{Results and Discussion}

Typical cyclic hardening curves determined in this study are shown in Figure 2, where stress amplitude is plotted as a function of accumulated plastic strain for modified and unmodified materials at two levels of plastic strain amplitude. This data shows that the surface modified material displays a higher stress amplitude beginning in the first few cycles, and continues to do so throughout the tests. An increase is observed of about $22 \mathrm{MPa}$ in saturation stress for the test at $6.6 \times 10^{-5}$ plastic strain, and $16 \mathrm{MPa}$ at $3 \times 10^{-4}$ plastic strain amplitude. Similar behavior was observed for all but the lowest and the highest strain ranges examined. No differences were noted in the amounts of accumulated strain required to achieve saturation. 
When cyclic stress strain curves are plotted for FCC single crystals, a plateau in the stress level is generally observed between plastic strains of $5 \times 10^{-5}$ and $5 \times 10^{-3}$, which is the range in which PSB's are thought to carry most of the plastic strain in constant plastic strain amplitude fatigue. Polycrystals are known to behave in a similar manner, with the plateau replaced by a region of positive but diminished

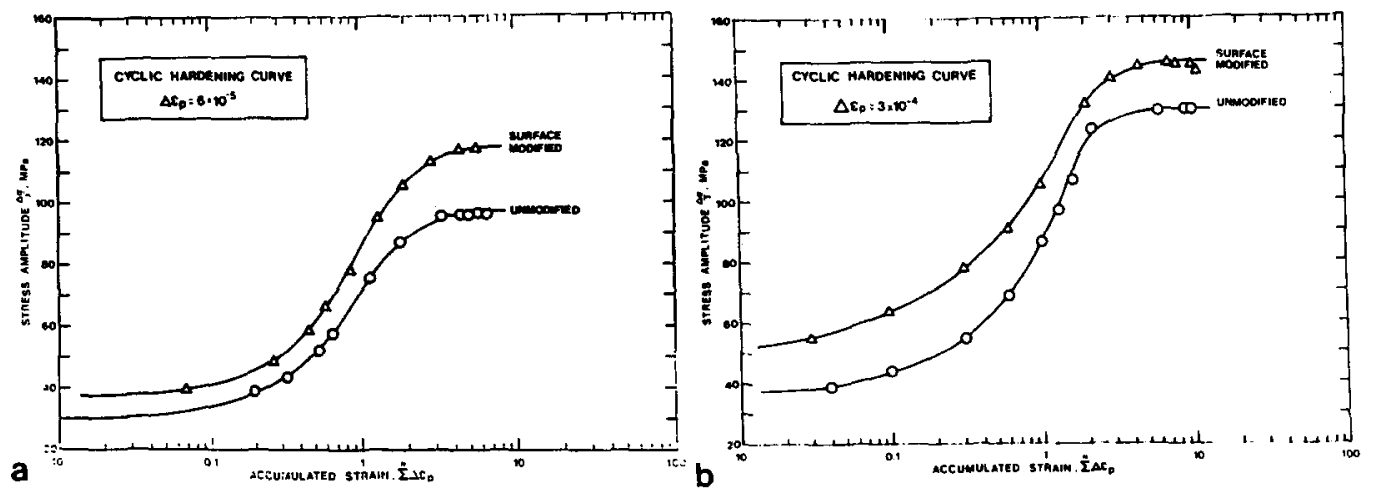

FIG. 2

Cyclic hardening curves for surface modified and unmodified specimens, a: plastic strain range $6.6 \times 10^{-5}$, b: plastic strain range $3 \times 10^{-4}$

slope over a similar interval of plastic strain ranges (16). The cyclic stress-strain (CSS) curves determined in the present study are shown in Fig. 3. An increase in saturation stress is apparent in the modified material and occurs over a broad range of plastic strain amplitudes between $5 \times 10^{-5}$ and $5 \times 10^{-3}$. Within this interval, the increase in stress is roughly constant at about $16 \mathrm{MPa}$. Both curves also display a shift to lower slope in this interval, and steeper slopes at both ends of each curve, behavior which is consistent with the operation of PSB's as the main carrier of plastic strain in this regime.

\section{FIG. 3}

Cyclic stress strain curves for implanted and un-implanted specimens showing saturation stresses as a function of the plastic strain range used in each test. The upper curve plots data for the surface modified aterial, and shows an increase in the saturation stress compared with the unmodified material at strain ranges between $5 \times 10^{-5}$ and $5 \times 10^{-3} 3$

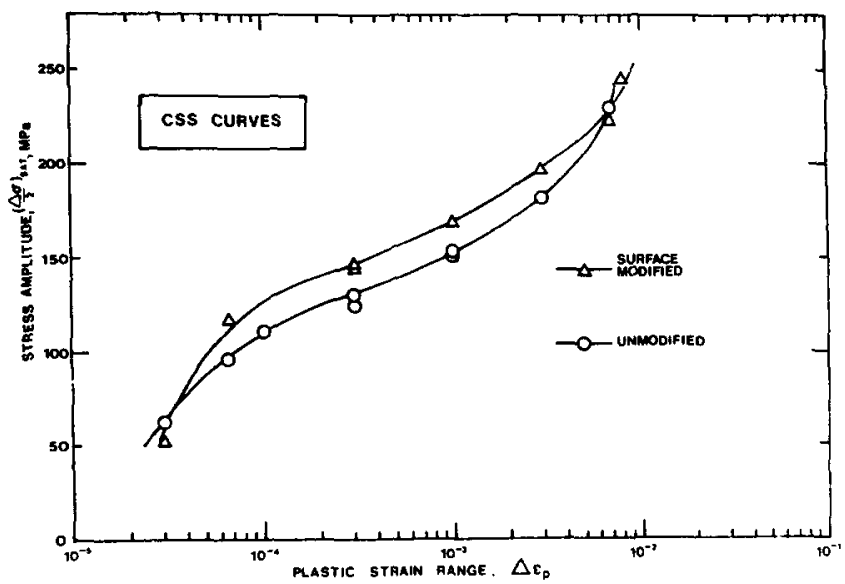

In addition to the mechanical behavior changes observed, we have seen striking changes in the evolution of surface morphology and in the rate and intensity of slip band emergence resulting from the ion beam surface modification. Figures $4 \mathrm{a}$ and $4 \mathrm{~b}$ show SEM micrographs of specimen surfaces taken from modified and unmodified samples which had identical strain histories. The lower density of persistent slip bands and the decrease in surface rumpling are readily apparent in the surface alloyed sample. Similar observations were obtained from other specimens, even those at the higher plastic strain ranges in which an increase in saturation stress was not clearly demonstrated. 
Figure 5 shows scanning electron micrographs taken from replicas of modified and unmodified specimens, both cycled at $3 \times 10^{-4}$ plastic strain. The replicas were made at total accumulated plastic strains of 2.0, 4.0 and 8.0 (the latter representing the material condition near saturation). From these and other observations, it is clear that the ion beam modified specimen shows a delay in the emergence of PSB's at the surface. In addition, the density of slip bands was found to be generally lower at the surface of the modified material when compared to the surfaces of the unmodified specimens.

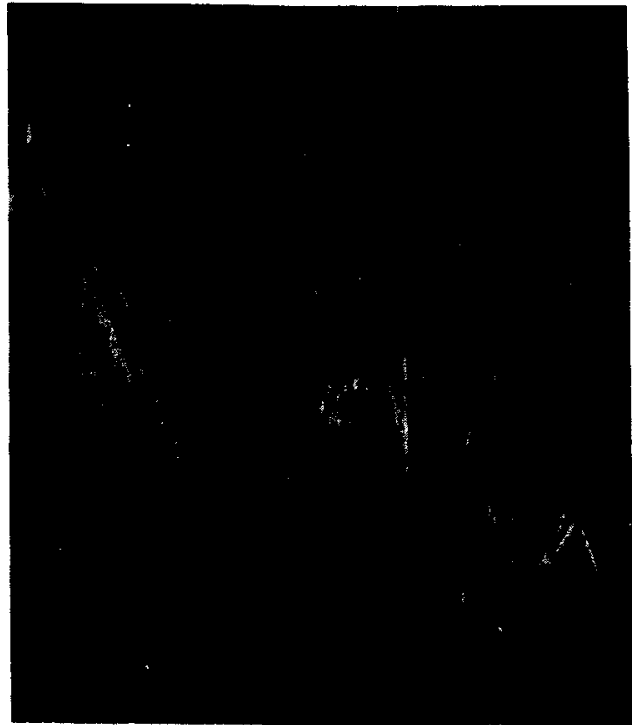

FIG. 4

Low magniffcation SEM micrographs of specimen surfaces at saturation; a: surface of ton bean modified nickel, b: surface of unmodified material. Specimens have identical strain histories: the plastic strain range is $3 \times 10^{-4}$

These results are believed to be significant, especially since the surface modification responsible for the observed changes in mechanical behavior is very shallow, probably not exceeding 100 to $200 \mathrm{~nm}$ in depth, and was applied to only $30 \%$ of the gage section surface area. If the implanted aluminum were to be distributed in the bulk, it would constitute an impurity on the order of only $30 \mathrm{ppm}$. Also, the effect of the surface treatment continued to be observed in tests at moderately high strain amplitudes, in which the surface suffered considerable accumulation of damage.

It is apparent that modification of a very shallow surface layer has produced changes in both bulk properties and in surface deformation behavior. While there is, as yet, no simple explanation for these results, some facts may be considered. First, and most obvious, is that the chemistry at the surface has been altered. Aluminum, acting as a solid solution strengthener, would increase the yield strength of the surface layer. Also, the stacking fault energy, which is high in pure nickel, would be reduced by alloying of the surface region, a change which could influence the development of slip line morphology (17). Secondly, a surface region exposed to energetic ion beam irradiation will acquire a high defect concentration, which would be expected to further increase the yield stress there. TEM observation of the near surface layer of nickel disks prepared by the same process used in the present study were found to have an extremely fine grain size (on the order of hundreds of nanometers) (18). It is reasonable to assume, then, that the modified surface layer possesses a much higher yield strength than the bulk, pure, nickel. If the surface yield stress is greater than that of the bulk, the plastic strain in the surface would be lower than in the bulk, and in fact, the surface plastic strain might remain at zero until a threshold stress amplitude was attained. It should also be pointed out that, unlike oxide layers, plated layers, or even diffusion layers, the ion beam mixed surface layer is continuous with respect to 
the substrate, and has no distinct interface, but rather a continuous gradient of composition and defect structure.

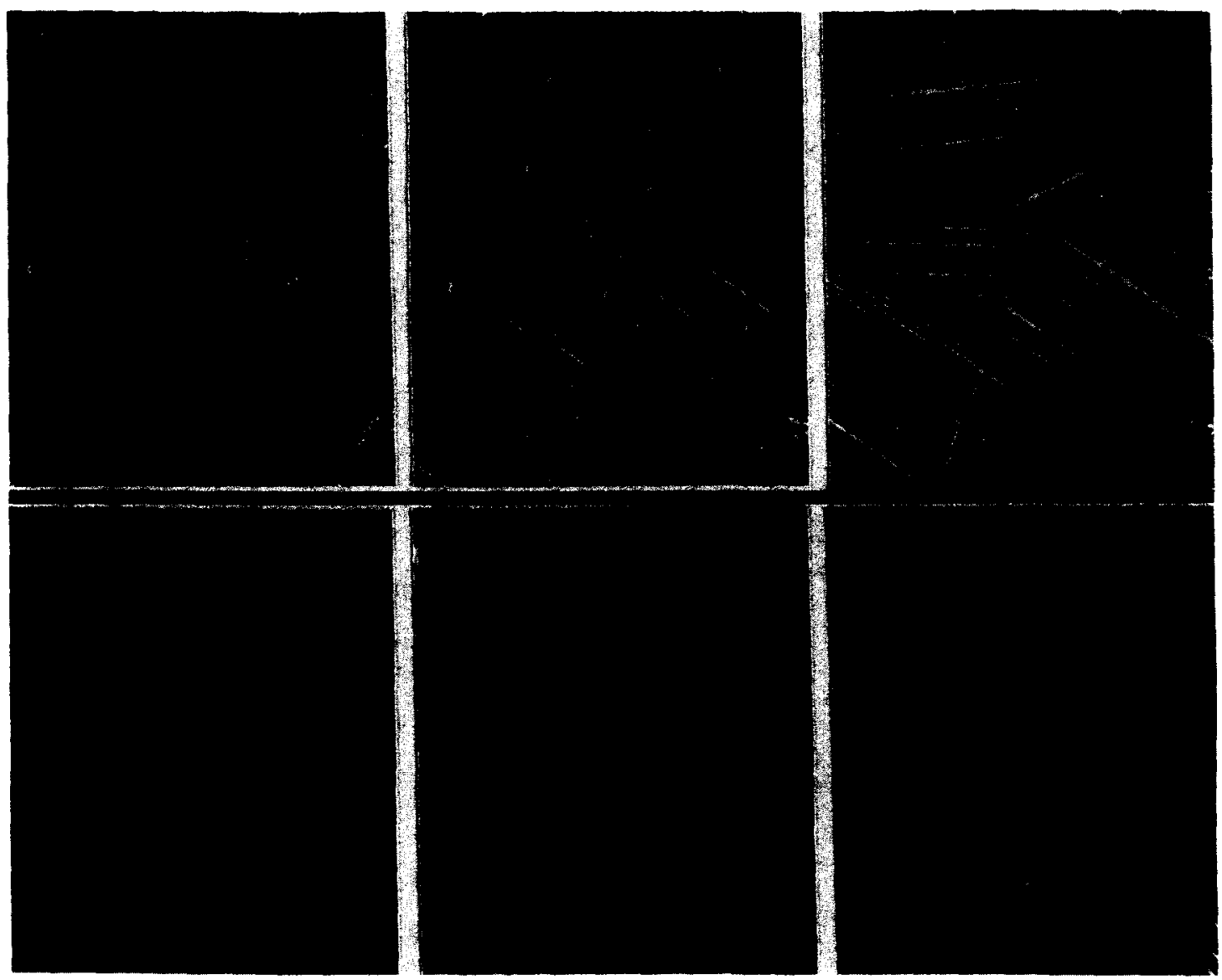

FIG. 5

SEM Micrographs of replicas made at progressive levels of plastic strain accumulation in two tests at $3 \times 10^{-4}$ plastic strain range. A-C: unmodified specimen; $D-F$ : ion beam modified specimen surface. Accumulated strains are $2.0,4.0$ and 8.0 respectively from left to right.

The experimental data show two distinct effects of the surface modification: the increase in saturation stress, and the inhibition of PSB extension to the surface. Changes in the yield strength and lowering of the stacking fault energy may help to account for the latter effect which may be associated mainly with changes in the properties in the near surface region. However, the observed increase in cyclic saturation stress, which is a change in behavior of the bulk material, suggests that the operation of surface dislocation sources has been influenced by the surface modification. Further study is under way, including the preparation of plated transverse section TEM foils, to determine what differences in near-surface and bulk dislocation substructure can be associated with the observed effects of ion beam surface modification in these specimens. 


\section{Conclusions}

1. The application of an ion beam mixed surface layer containing approximately 25 at.\% aluminum increases the cyclic saturation stress of pure polycrystalline nickel by approximately 15 to $20 \mathrm{MPa}$ when cycled at plastic strain ranges between $5 \times 10^{-5}$ and $5 \times 10^{-3}$. At any given level of accumulated strain, the stress amplitude of the surface modified material is greater than for an unmodified material tested under the same conditions.

2. The emergence of persistent slip bands at the surface is delayed by the surface modification, and at saturation, the surface modified material shows a lower density of PSB's and a lesser degree of surface rumpling when compared to an unmodified material.

\section{Acknowledgements}

This research was funded by the National Science Foundation under grant \#DMR8310032. The authors gratefully acknowledge the contribution of J. Eridon and G. S. Was of the Department of Nuclear Engineering at the University of Michigan, and Lynn Rehn, of the Argonne National Laboratory Tandem Accelerator Facility.

\section{$\underline{\text { References }}$}

1. G. S. Was and R. M. Pelloux, Met. Trans. 10A, 656 (1979).

2. L. H. Berck, C. P. Sullivan and C. H. Wells, Met. Trans. 1, 1595 (1970).

3. K. Y. Chen and E. A. Starke Jr., Mat. Sci. Eng. 24, 209 (1976).

4. W. Hu, C. R. Clayton and H. Herman, Scripta Met. 12, 679 (1978).

5. A. Kujore, S. B. Chakrabortty and E. A. Starke, Proc. Symp. Mat'ls Res.Soc., Cambridge, Mass., 1979. Preece, Hirvonen, Eds. AIME, p132 (1980).

6. A. W. Sleeswyk, H. J. Kok and G. Boom, Scripta Met. 14, 919 (1980).

7. K. Jata, J. Han, E. Starke and K. Legg, Scripta Met. 17, 479 (1983).

8. K. Jata and E. Starke, J. Met. Aug. 1983 p23.

9. K. Hohmuth, E. Richter and B. Rauschenbach, Mat. Sci. Eng. 69, 191(1985).

10. H. Mughrabi, Scripta Met $/ 33,479$ (1978).

11. C. Laird, Metallurgical Treatises, USA-China Bi-lateral Conference of the AIME, Beijing. Elliot, Tien, Eds.

12. K. V. Rasmusșen and O. B. Pedersen, Acta Met. 28, 1467.

13. Z. S. Basinski and S. J. Basinski, Scripta Met. 18, 851 (1984).

14. Z. Basinski, R. Pascual and S. Basinski, Acta Met. 31, 591 (1983)

15. L. M. Brown Met. Sci. Aug/Sep. 1977 p.315.

16. O. Pedersen, K. Rasmussen and A. Winter, Acta Met. 30, 57 (1982)

17. H. Neuhauser, Dislocations in Solids, F. Nabarro, Ed. North-Holland, 1983 p321.

18. J. Eridon, the University of Michigan Dept. of Nuclear Engineering (private communication). 